\title{
An Improved Tabu Search Algorithm Based on Grid Search Used in the Antenna Parameters Optimization
}

\author{
Di He and Yunlv Hong \\ Shanghai Key Laboratory of Navigation and Location-Based Services, Shanghai Jiao Tong University, Shanghai 200240, China \\ Correspondence should be addressed to Di He; dihe@sjtu.edu.cn
}

Received 23 September 2014; Revised 29 January 2015; Accepted 6 February 2015

Academic Editor: Hsuan-Ling Kao

Copyright (C) 2015 D. He and Y. Hong. This is an open access article distributed under the Creative Commons Attribution License, which permits unrestricted use, distribution, and reproduction in any medium, provided the original work is properly cited.

In the mobile system covering big areas, many small cells are often used. And the base antenna's azimuth angle, vertical down angle, and transmit power are the most important parameters to affect the coverage of an antenna. This paper makes mathematical model and analyzes different algorithm's performance in model. Finally we propose an improved Tabu search algorithm based on grid search, to get the best parameters of antennas, which can maximize the coverage area and minimize the interference.

\section{Introduction}

In mobile communication systems, such as the current $2 \mathrm{G}$ and $3 \mathrm{G}$ networks, small cells divided by the base stations are used to cover the entire region. So the base station should have the best possible coverage area in order to improve the quality of service (QoS). As to base station antenna, the most important factors that affect the cell coverage are antenna's azimuth angle, vertical down angle, and transmit power. As to the terminal, we think there are two targets which have to be considered. They are RSRP (Reference Signal Receiving Power) and SINR (Signal to Interference plus Noise Ratio) [1]. The RSRP characterizes the average signal power obtained by the mobile terminal which depends on the power antenna transmits, distance between base station and terminal, and direction of terminal to the base station, and so forth. SINR is different from the noise in the propagation path such as AWGN (Additive White Gaussian Noise). It focuses on the interference from different signals which lead to the decline in SNR (Signal-to-Noise Ratio). SINR mainly originates from the interference caused by the signals from the same base station to different terminals and from different base stations to one terminal. So we should choose the right antenna parameters to maximize the RSRP and minimize the SINR.

Recent antenna parameter adjustment algorithms are mainly based on experience and manual adjustment. Besides, we can use some optimization algorithms, such as Powell search algorithm, and intelligent algorithms such as GA (Genetic Algorithm) [2], TS (Tabu Search) [3, 4], IWO (Invasive Weed Optimization) [5, 6], PSO (Particle Swarm Optimization) [7-9], SO (Stochastic Optimization) [10], and BC (Bee Colony Algorithm) [11]. Manual adjustment consumes a large amount of time. We can make predictions by Powell search algorithm, GA, TS, and some other optimization algorithms, but there are also some limitations. In a practical scene, it is actually an NPC (NP-complete) problem to get best RSRP and SINR by adjusting azimuth angle, vertical down angle, and transmit power. If we try to get the result by exhaustive attack method, the algorithm will have exponential complexity. Powell algorithm can easily get a local optimal solution, but it is easy to fall into a local extreme trap. Otherwise, intelligent algorithms such as GA, TS, IWO, PSO, SO, and BC algorithms are global algorithms. But the problem of GA algorithm is that it does not guarantee that the revolution direction of next generation is the best one. This leads to the excessive number of iterations. TS algorithm has similar problem to GA. IWO algorithm converges prematurely sometimes, and it is easy to fall into the local optimization. PSO algorithm does not perform very effectively to solve some discrete optimization problems, and it is also easy to fall into the local minimum or local optimization. As to the $\mathrm{SO}$ and $\mathrm{BC}$ algorithms, the convergence speeds are hard to be guaranteed. 


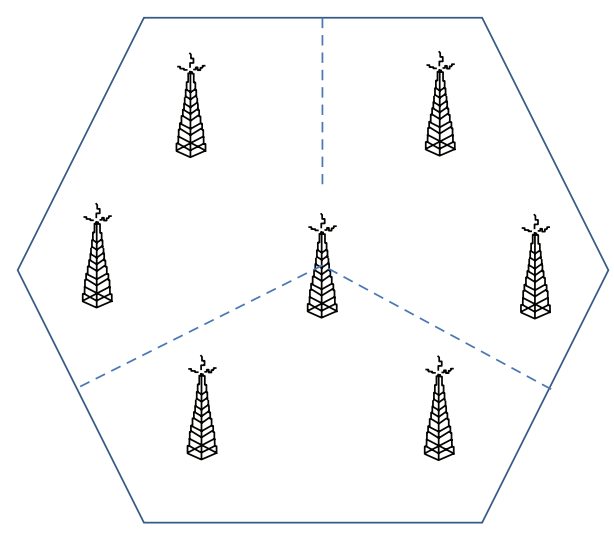

Figure 1: A typical cellular network unit.

In this study, we propose an improved TS algorithm based on Grid Search to solve the problem of antenna parameter adjustment and optimization. By combining both the TS algorithm and the Grid Search strategy, the advantages of both approaches can be maintained and developed. At the same time, it can be found that the time consuming of the optimization process can be reduced efficiently, and the final optimization results guarantee that it can get the global optimum, compared with that of the exhaustive attack method (EAM) in the computer simulations.

This paper mainly discusses the application of intelligent TS algorithm in antenna parameters optimization problem. By using Tabu Search algorithm based on grid search and changed-pace one-dimensional search, we can get a global extreme in short time. Finally, we use multicell joint adjustment to get a better cell coverage. Section 2 gives the practical scene in this paper. Section 3 describes the corresponding mathematical model. Section 4 introduces the proposed improved algorithm. Section 5 gives the computer simulation results, and Section 6 is the conclusion.

\section{Scene Model}

In modern mobile communication system, we often use cell coverage to solve the problem of signal coverage of the whole system. The entire region is divided into several cells according to antenna of base station. The terminal directly communicates with the base which it belongs to. The cellular network is a typical model of cell coverage [12]. As shown in Figure 1, in a typical cellular network unit, every base has three antennas. The direction between every two figure is 120 degrees. One cell is divided into three sectors, and a base covers a rhombus shape area. The current mobile systems, such as 3G CDMA 2000, WCDMA network, and latest 3GPP LTE, all use the similar three antennas coverage model.

But the shape of the base coverage as well as the number of sectors is adjusted with the actual needs in practical engineering. As shown in Figure 2, this is an actual base station scene in certain area in Norway. The black point in figure represents the location of base station. The arrow represents the direction of base antenna. The distribution of base stations and directions of antennas have no rules to

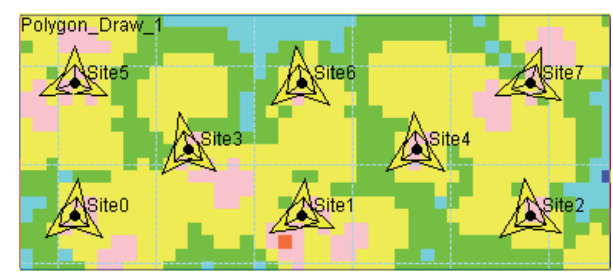

Figure 2: A base station distribution scene in Norway.

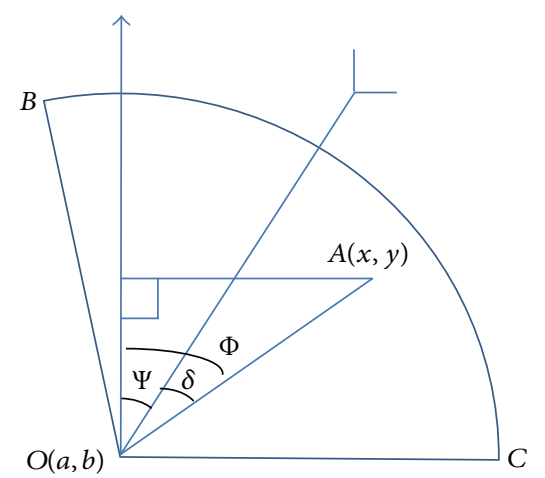

Figure 3: Antenna's horizontal angle.

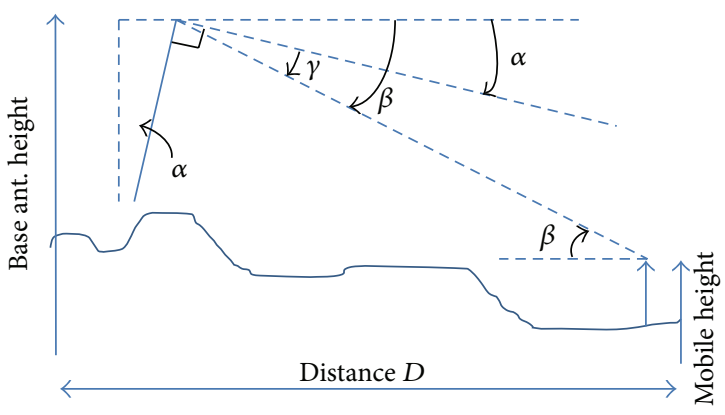

FIgURE 4: Antenna's vertical angle.

follow. We can see the base in the bottom of the figure, the base station only has 1 to 2 sectors. This figure illustrates that in practical engineering, it is difficult to use typical cellular network.

As the distribution of base stations and directions of antennas have no rules to follow, we can only adjust antennas parameters to maximize the coverage area. To simplify the mathematical model, in practical engineering we just consider three major parameters. They are antenna's azimuth angle $\psi$, vertical down angle $\gamma$, and transmit power $C$. Here, transmit power $C$ means the transmit power on reference signal subcarriers. The azimuth angle and vertical down angle are shown in Figures 3 to 5 .

Figure 3 is the top view of a sector. Sector $B O C$ is coverage area. $O$ shows the location of base station. $A$ shows the location of moving terminal and we assume the location of terminal is definite. Angle $\phi$ shows the horizontal angle between terminal and base station. Angle $\psi$ is the azimuth angle (reference to true north). Angle $\delta$ shows the direction between projection from vertical down angle to 


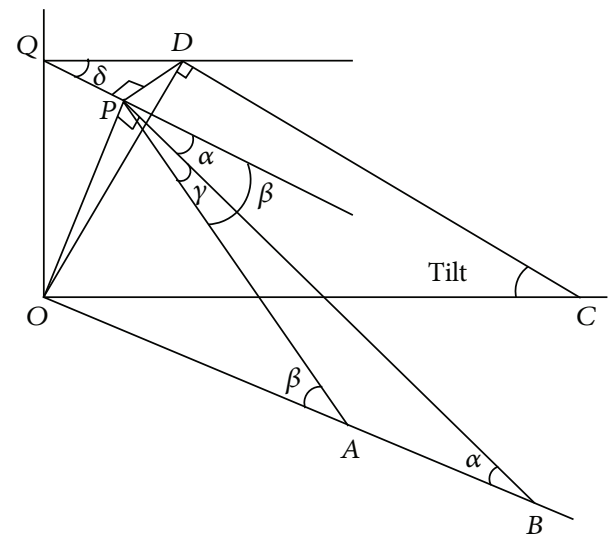

FIgURE 5: 3D figure of antenna.

ground and moving terminal. As we can get the horizontal directional antenna gain by using $\psi$ to check it from the form provided by base equipment producer, we consider $\psi$ to be an independent variable in the optimization algorithm.

In Figure 4, $\alpha$ is declination of the antenna (more details can be found in Figure 5). $B$ is the elevation angle from terminal to base antenna. $\gamma$ shows the vertical down angle. Based on the same reason in last paragraph, we also consider $\gamma$ to be an independent variable in the optimization algorithm.

\section{Mathematical Model}

3.1. The Structure of the Objective Function. Assume $\Omega=$ $\left\{C_{1}, C_{2}, \ldots, C_{n}\right\}$ is the solution space of all states. $C_{i}(i=$ $1,2, \ldots, n)$ represents a configuration of one sector's parameters. It consists of three parameters $(x, y$, and $z)$. Here, $x, y$, and $z$, respectively, represent antenna's azimuth angle, vertical down angle, and transmit power. So our goal is to obtain an optimal solution of the solution space, which makes the antennas parameters expressed as in the following formula:

$$
(\underbrace{\left\{x_{1}, y_{1}, z_{1}\right\}}_{C_{1}}, \underbrace{\left\{x_{2}, y_{2}, z_{2}\right\}}_{C_{2}} \cdots \underbrace{\left\{x_{n}, y_{n}, z_{n}\right\}}_{C_{n}}) \text {. }
$$

While considering the coverage performance, both the coverage area of the whole region and interference from other bases should be taken into account. In communication field, RSRP and SINR can be an index. When at a point in the scene, RSRP is over certain threshold and SINR is below certain threshold; this point is covered by the base successfully. And then accounting the successfully covered area in the whole region, if the ratio reaches the maximum, we get a global solution of the cell coverage problem. The objective function is defined by the following formula:

$$
f=k_{1} * A+k_{2} * B .
$$

In the above formula, $f$ represents the objective function. $A$ shows the ratio of area whose RSRP exceeds the threshold and area of whole region. $B$ shows the ratio of area whose SINR exceeds the threshold and area of whole region. Here, $k_{1}$ and $k_{2}$ are weighting constants and $k_{1}+k_{2}=1$.

When the objective function gets best solution $f_{\text {best }}$, we can take the best solution

$$
(\underbrace{\left\{x_{1 b}, y_{1 b}, z_{1 b}\right\}}_{C_{1}}, \underbrace{\left\{x_{2 b}, y_{2 b}, z_{2 b}\right\}}_{C_{2}} \cdots \underbrace{\left\{x_{n b}, y_{n b}, z_{n b}\right\}}_{C_{n}})
$$

to decide the actual parameters in antennas adjustment.

3.2. Calculation of RSRP and SINR. According to cost231hata propagation model, we define RSRP and SINR as follows. Terminal's RSRP calculation formula is as shown:

$$
A(d B m)=\max _{0 \leq i \leq J-1}\left(P_{\mathrm{RS} \_\mathrm{RE}}^{i}-L_{\mathrm{DL}}^{(i, u)}\right) .
$$

In formula (4), $P_{\mathrm{RS} \_\mathrm{RE}}^{i}$ is the transmit power of sector $i$ on reference signal on subcarrier. $J$ is the total number of cells which need to be considered in the network. $L_{\mathrm{DL}}^{(i, u)}$ is the loss from antenna to the terminal $u$. And we have $L_{\mathrm{DL}}^{(i, u)}(d B)=$ equipment loss + propagation path loss - the base station antenna gain. According to Section 2, antenna gain is decided by antenna's azimuth angle and vertical down angle. So the first item $A$ of objective function $f$ is decided by $\psi, \gamma$, and $C$.

Assuming that the transmit power is distributed in all of a reference signal, the terminal SINR calculation can be written as

$$
B(d B)=A-10 \times \lg \left(I_{\mathrm{DL}}^{(\mathrm{total}, u)}+P_{\text {Noise_RE }}\right) .
$$

In formula (5), $I_{\mathrm{DL}}^{(\mathrm{total}, u)}=\sum_{j=1}^{J} 10^{I_{\mathrm{DL}}^{(j, u)} / 10}$ represents the total downlink interference. $J$ is the total number of cells that need to be considered in the network. $I_{\mathrm{DL}}^{(j, u)}(\mathrm{dBm})=P_{\mathrm{RE}}^{j}-$ $L_{\mathrm{DL}}^{(j, u)}$ represents the interference from sector $j$ to terminal $u$. $P_{\text {Noise_RE }}$ is the noise power on a reference signal.

\section{Improved Tabu Search Algorithm Based on Grid Search}

4.1. Algorithm Selection. As previously mentioned, solving objective function is an NPC problem, because its complexity grows exponentially with the increase in the number of sectors. We cannot use a polynomial to describe the function, so any algorithm based on derivative cannot be used in this paper. In practical engineering, we often use some algorithm independent of derivative such as Powell search algorithm. Powell search does not require the objective function's derivative, and the iterative process is relatively simple. However in this scene model, considering antenna's adjustable minimum scale, Powell's search direction will lose conjugate after several rounds. In practical engineering, we firstly optimize the RF parameters with continuous range of values. Secondly we take the nearest discrete value to be the optimization result. But Powell search also has a problem that it is easy to fall into the local extreme trap. The optimization result is not really a global solution. 


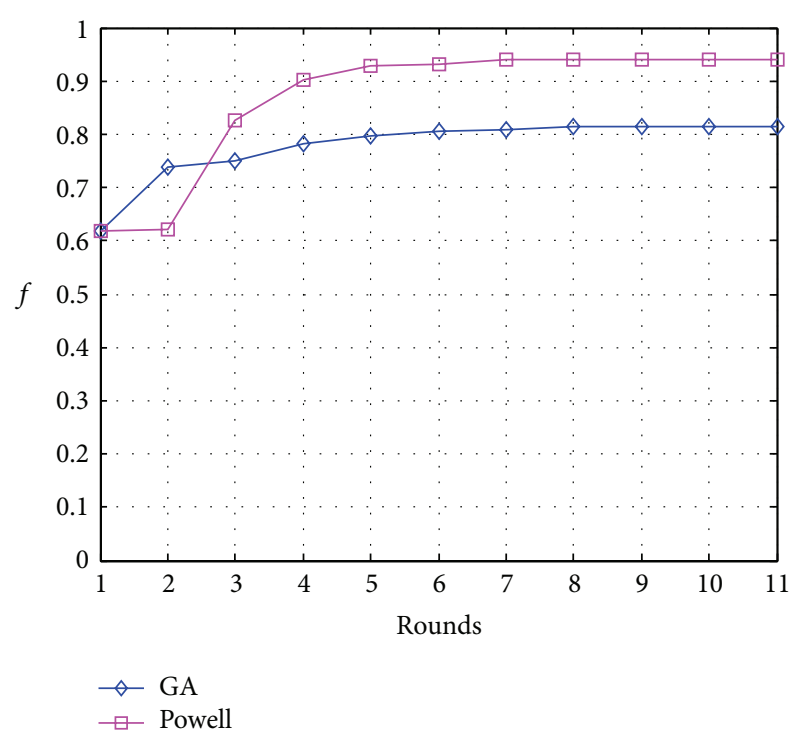

FIGURE 6: Partly efficiency comparison between GA and Powell algorithm.

Intelligent algorithm can also be used in this scene such as Genetic algorithm which is widely used in solving engineering problems $[13,14]$. Compared with Powell search, intelligent algorithm can effectively solve the global minimum, but the convergence speed is rather slow. To verify this problem, we select the base station scene in a practical engineering to make simulation. As shown in Figure 6, Genetic algorithm has obvious drawback of slow lifting speed. Generally it consumes several times iterations to Powell search to get the same degree of convergence.

In Figure 7, it shows that after 60 iterations the final objective function reaches 0.963 , and the Powell search algorithm has a result of 0.953 . Although GA algorithm has improved relative to Powell search, the improvement is not significant. The above figure confirms the conjecture that the lifting speed of GA is slower than that of Powell algorithm. That is because GA does not guarantee that the revolution direction of next generation is the best one. The reason why final improvement of GA is not significant is the limitation of iteration counts. After an infinite number of iterations, we could get the global extreme by GA algorithm.

By analyzing the actual performance of intelligent and nonintelligent algorithm, we can get he following conclusion. (1) Nonintelligent algorithm like Powell search has fast convergence speed; (2) nonintelligent algorithm like Powell search is easy to fall into local extreme trap; and (3) GA's lifting speed is too slow.

Based on the above conclusions, we choose another intelligent algorithm, which is the Tabu Search. The reason to select TS in solving this engineering problem depends on the following facts. Firstly, as an intelligent algorithm, TS can avoid local extreme trap and reach the global optimization [15]. Secondly, a large number of studies have shown that the TS algorithm is applicable to noncontinuous problems, which fits the circumstance of the antenna parameter adjustment

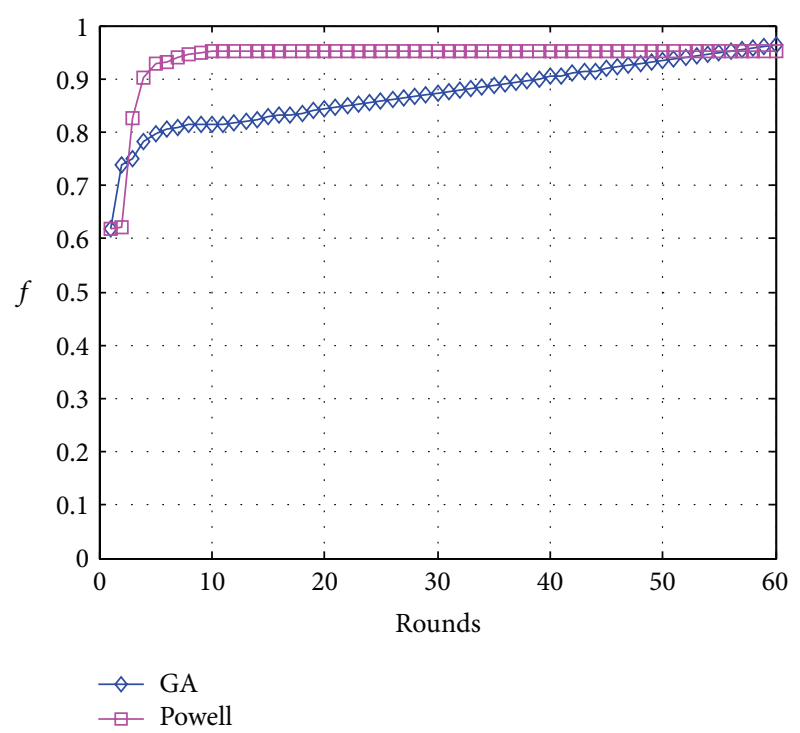

FIGURE 7: Completely efficiency comparison between GA and Powell algorithm.

problem well [16]. Thirdly, TS algorithm can construct neighborhood and candidate freely, which makes it possible to improve the convergence speed forward to the optimal solution. TS can effectively combine the advantages of both algorithms, avoiding their disadvantages. The basic Tabu Search algorithm is shown in Algorithm 1.

4.2. Local Selection Algorithm. As shown in Algorithm 1, the main idea of TS algorithm is to quickly get a local extreme. Then put the local extreme into Tabu list and find the next one. This algorithm requires very fast local search speed, so this paper uses changed-pace one-dimensional search on a grid shape candidate field. Following are the details.

The solution of the scene should be as the form of (1). Sector $i$ corresponds to a set of parameters $\underbrace{\left\{x_{i}, y_{i}, z_{i}\right\}}_{C_{i}}$. If we view $x_{i}, y_{i}$, and $z_{i}$ as coordinates in three-dimensional space, when this set of parameters of sector $i$ search local extreme in its neighborhood, the candidate field forms a grid shape.

In a grid search problem, we can consider that the search direction is the direction of the initial point to its near point. Then, if we just consider the parameters of azimuth and vertical angle, it should be a two-dimensional grid. And there are 8 search directions that are up, down, left, right, top-left, bottom-left, top-right, and bottom-right. In this scene model, it should be a three-dimensional grid and there are 26 search directions.

After determining the search direction, we can get a maximum in every search direction by calculating objective function. Theses local maximum points form a new candidate field. Choose the biggest in the field. If it is not in the Tabu list, put it into Tabu list and it becomes next round's initial point. If it is better than the "best solution" in the list, it becomes the new "best solution." If the improvement of objective function 


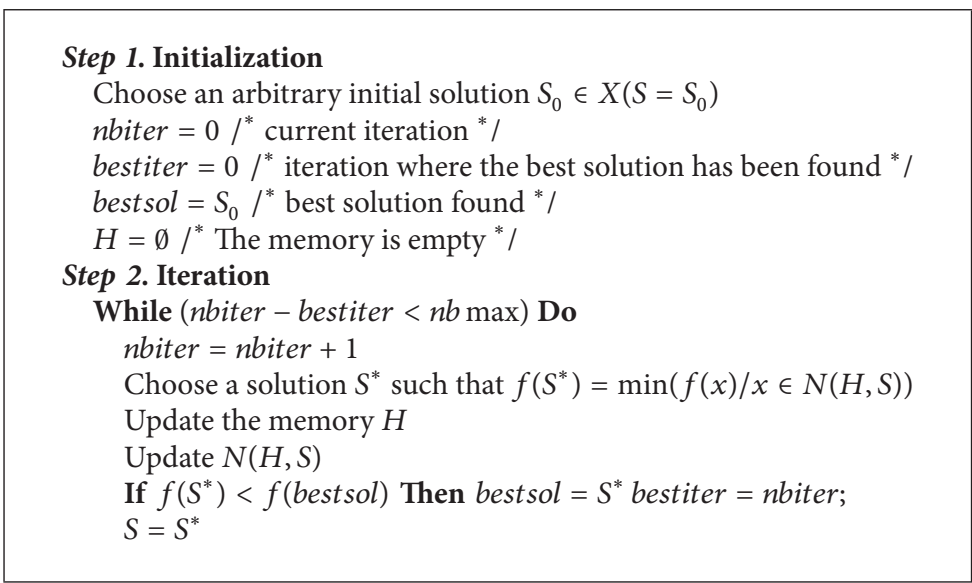

Algorithm 1: Basic Tabu search algorithm.

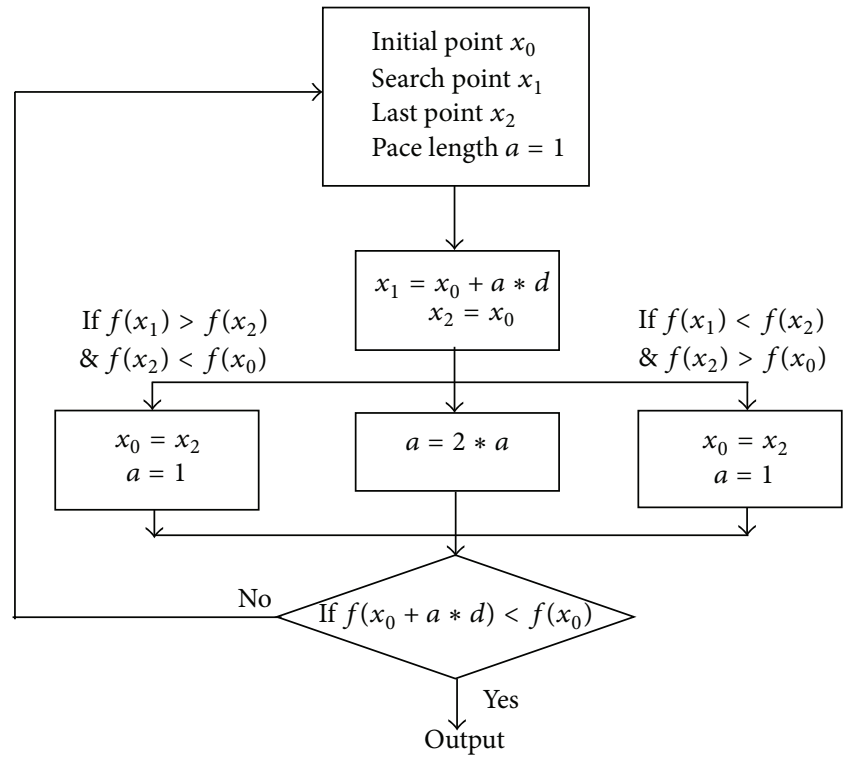

Figure 8: The process of changed-pace one-dimensional search.

$f$ is smaller than $\varepsilon$, the current "best solution" becomes the global solution.

Searching the maximum on one direction, we use changed-pace one-dimensional search. Intelligent adjustment step makes the decrease in the number of searches to improve the algorithm speed. The process is shown in Figure 8.

In Figure 8, $d$ is the search direction. $f(\cdot)$ is the objective function. $a$ is the pace length. If it fails to get feasible solution in on direction, the system doubles the pace length $a$. If it succeeds to get a feasible solution, the system decreases the pace length and carefully searches for feasible solution's near points.

Besides, in the local extreme search, we can record the objective function of one point which has been searched. Next time the algorithm reaches this point, we do not need to calculate the objective function again.

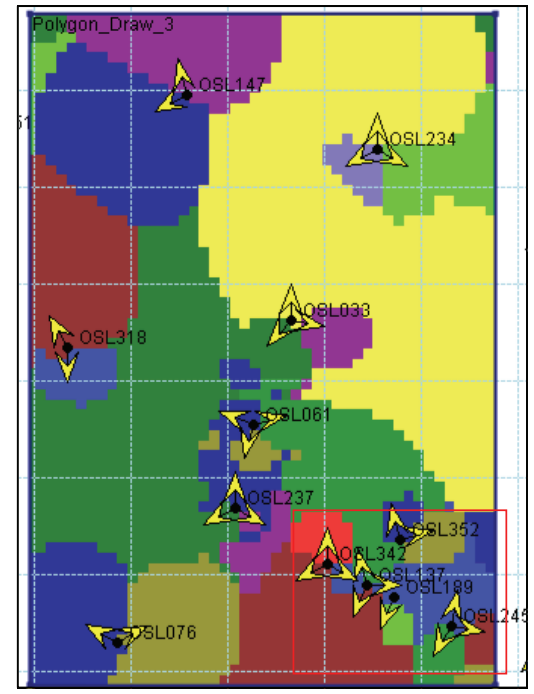

FIgURE 9: Another base station distribution scene in Norway.

4.3. Multicell Adjustment. In above algorithm, the system calculates from sector 1 to $n$ repeatedly to refresh the current best solution of (1).

This loop calculation has two problems: (1) the calculation of sector $i$ affects the result of sector $i-1$ and (2) in practical engineering, because of irregular distribution of base stations, multicell adjustment is more efficient. As shown in Figure 9.

In Figure 9, the base stations in red box concentrated in a small area. If their parameters are adjusted separately, they will interfere with each other. But if we adjust their parameters together, we do not need to modify them again when we adjust another area's base stations in next round. Like the station OSL076 in the bottom-left, it is better to be adjusted individually.

Considering the efficiency of algorithm, multicell adjustment consumes more time in every one round, but the performance also get surprising improvement in one round. For performance comparison, we also use the IWO optimization approach in [5] and the PSO optimization approach in [7] 


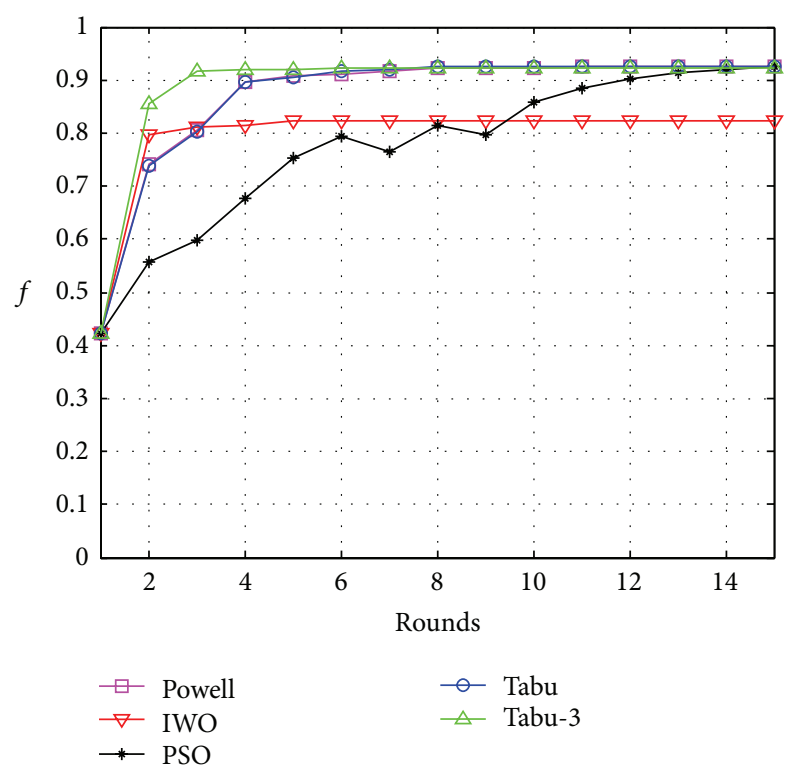

FIGURE 10: Efficiency comparison between three algorithms.

to solve the same problem. As shown in Figure 10, the lines "IWO" and "PSO" denote the IWO and PSO approaches, respectively; the line "Tabu" represents single-cell adjustment improvement curve with round number, and the line "Tabu3 ” represents three-cell adjustment improvement curve.

In Figure 10, we can get that, under the same rounds, the objective function lifting rate with multicell adjustment is much higher than that with single-cell adjustment. The IWO approach has a rapid convergence speed but it falls into a local minimal trap and cannot escape. And the PSO approach converges slower than other methods and it may undergo some gurgitations during the optimization process. In this study, we use MATLAB to make simulations. The time consumption of multicell adjustment is larger than the singlecell's. But considering MATLAB platform itself is suitable for matrix operations and not good at iteration calculation. When we run the algorithm on the $\mathrm{C}$ platform, multicell adjustment can further reduce the run time. As shown in Figure 11.

In Figure 11, we can see time consumption of multicell adjustment decreasing dramatically on C platform. However, single-cell adjustment has not this feature.

In the process of algorithm, the order of optimization is decided by the division of bases' group. We define bases close on the distance being in the same group, and the details are as follows.

(1) Firstly choose the top-left base to be the initial base and choose the base which is near to initial base to be one group.

(2) Optimize the parameters in this group; the order of sector optimization is random.

(3) After optimizing the parameters, record the cells which have been optimized in the list.

(4) Choose the base which is the nearest to this group, but which has not been optimized, to be the next initial

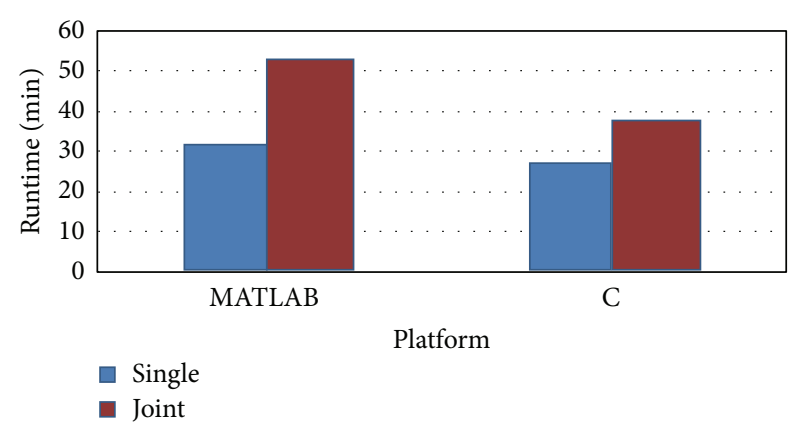

FIGURE 11: Time consuming of two adjustment schemes in different platform.

base. Choose the base which is near initial base to be one group.

(5) If the added base has been in the optimized list and its distance to initial base is over some threshold, throw this base.

(6) Repeat this process until all the bases are optimized.

\section{Computer Simulation Results}

5.1. Simulation Experiment. We randomly choose 5 actual base scenes in Norway and separately use Powell and improved TS algorithm to make simulation and account their results. Table 1 shows the detailed parameters of the experiment.

Then we can get result as shown in Figure 12.

As shown in Figure 12, the performance of improved TS algorithms (red and blue lines) is better than the Powell algorithm (yellow line). And applying multicell algorithm, the objective function of some scene get further improved. However, we can also see that the multicell algorithm has its limitations. In scene 1 (Figure 2), the distribution of base stations is relatively sparse, the performance of multicell adjustment is not much better than single-cell but wastes more time. In this case, single-cell adjustment is a good choice. In scene 2 (Figure 9), when the base stations concentrated in a small area, multicell adjustment is better choice. But from all randomly selected five scenes' results, joint adjustment's performance is better than the single one's.

5.2. Robustness Test. Robustness refers to reliability of the system. When some accident occurs, the system should still work. In our paper, when adjusting the antenna's azimuth and vertical angles in practical engineering, the adjusted error will lead to deviation between actual result and theoretical value. So we have to consider the change of SINR and RSRP here.

In engineering, we cannot adjust the antenna's parameter very accurately. For example the calculated angle should be 120 degrees, but the actual adjusted result may be 120.5 degree. Robustness test requires that, under such deviation, the system still have a stable performance.

Now we set the optimized result to be the benchmark. Randomly change the parameters including azimuth and 
TABLE 1: Experiment parameters.

\begin{tabular}{lccc}
\hline Tabu list length $(l)$ & Multicell number $(n)$ & Aspiration criterion & Max iteration number \\
\hline 5 & 3 & Tabu list is full & 10 \\
\hline Search ace length $(d)$ & TS adjustment law & Propagation & Number of $k_{1}, k_{2}$ \\
\hline 1 & Single/multicell joint & Cost231 & $k_{1}=k_{2}=0.5$ \\
\hline
\end{tabular}

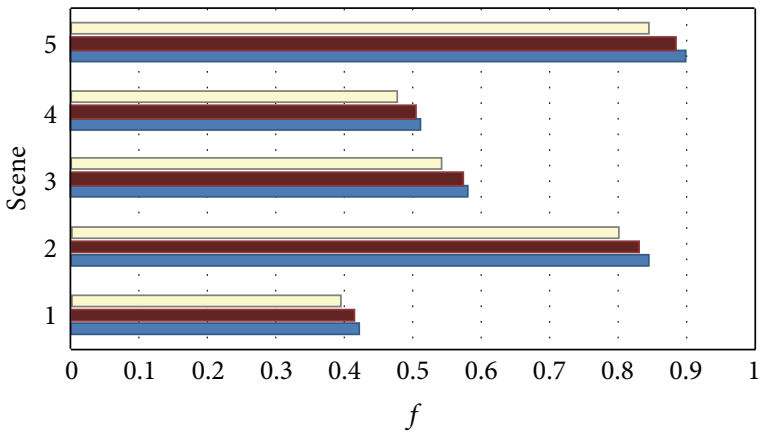

$\square$ Powell

- Grid TS

- Joint TS

FIGURE 12: Result comparison with five scenes.

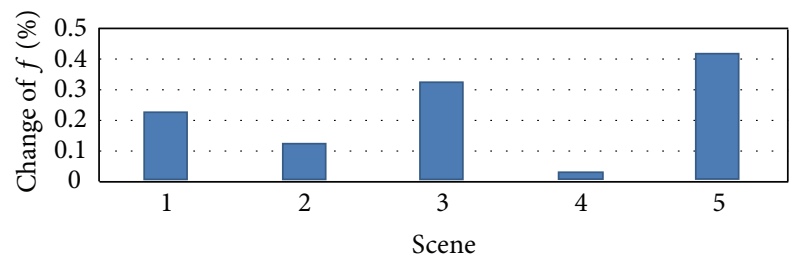

FIGURE 13: Robustness test results.

vertical down angles. The error range is up to 1 degree, but in fact the practical deviation in engineering is less than 1 degree.

As shown in Figure 13, the robustness test shows that when changing from 4 to 8 parameters, the change of objective function is less than $0.5 \%$. Considering the actual deviation is less than 1 degree, the performance degradation caused by the error is much smaller. So we can say out proposed algorithm is very strong through this robustness test.

5.3. Optimal Solution Test. As the antenna parameter adjustment is an NPC problem, we cannot prove that we can obtain the optimal solution in theory. Now we make simulation to prove that our optimized result is very near to the optimal solution. We choose a relatively small scene (scene 3 ) and use exhaustive attack method to get the optimal solution. Exhaustive attack method's complexity is exponential complexity, and it consumes much more time than the TS algorithm. At the same time, we also introduce the IWO optimization approach in [5] and the PSO optimization approach in [7] for comparison.

In Figure 14, the objective function value of improved TS algorithm is 0.583 , and the value of exhaustive attack method

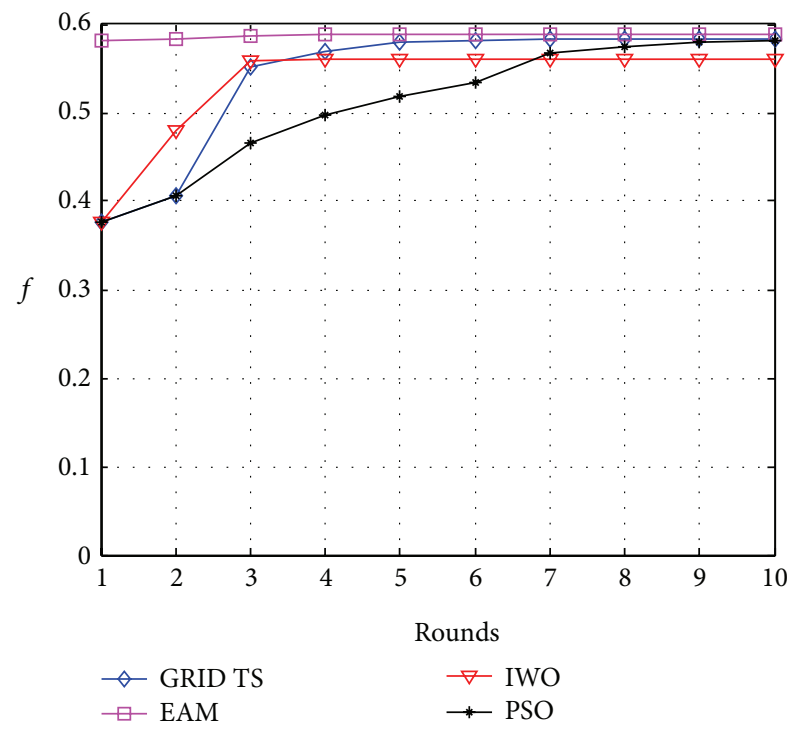

FIgURE 14: Exhaustive method test.

(EAM) is 0.588 . This experiment proves that our algorithm's result is very near to the optimal result. The proposed TS search succeeds to get a global extreme in a short time. It is very suitable for the practical engineering. Simultaneously, it can be found that the IWO approach and the PSO approach also have the same problem as in Figure 10.

\section{Conclusions}

This paper discusses how to get a set of parameters of base antenna to improve the coverage of the whole region. By building up the corresponding scene model and mathematical model, we propose an improved TS search algorithm and compare it with some other algorithm to analyze its advantage. We make robustness test to prove it can work well when accident occurs. This means the algorithm is very strong. We make optical test to prove our algorithm can get a global extreme and not easy to fall into local extreme trap. Besides, we propose a multicell joint adjustment process by analyzing the base distribution. The proposed approach can also be utilized in other application scenes, such as the indoor wireless positioning or localization and indoor visible light communications.

\section{Conflict of Interests}

The authors declare that there is no conflict of interests regarding the publication of this paper. 


\section{Acknowledgments}

This research work is supported by the National High Technology Research and Development Program of China under Grant no. 2013AA013602 and jointly funded by the Beidou Navigation Satellite System Management Office (BDS Office) and the Science and Technology Commission of Shanghai Municipality under Grant no. BDZX005.

\section{References}

[1] C. Fan, Principles of Communications, 1991.

[2] G. Ewald, W. Kurek, and M. A. Brdys, "Grid implementation of a parallel multiobjective genetic algorithm for optimized allocation of chlorination stations in drinking water distribution systems: chojnice case study," IEEE Transactions on Systems, Man and Cybernetics Part C: Applications and Reviews, vol. 38, no. 4, pp. 497-509, 2008.

[3] F. Glover, "Tabu search-part I," ORSA Journal on Computing, vol. 1, no. 3, pp. 190-206, 1989.

[4] F. Glover, "Tabu search-part II," ORSA Journal on Computing, vol. 2, no. 1, pp. 4-32, 1990.

[5] F. M. Monavar, N. Komjani, and P. Mousavi, "Application of invasive weed optimization to design a broadband patch antenna with symmetric radiation pattern," IEEE Antennas and Wireless Propagation Letters, vol. 10, pp. 1369-1372, 2011.

[6] Z. D. Zaharis, C. Skeberis, T. D. Xenos, P. I. Lazaridis, and J. Cosmas, "Design of a novel antenna array beamformer using neural networks trained by modified adaptive dispersion invasive weed optimization based data," IEEE Transactions on Broadcasting, vol. 59, no. 3, pp. 455-460, 2013.

[7] C. Li, S. Yang, and T. T. Nguyen, "A self-learning particle swarm optimizer for global optimization problems," IEEE Transactions on Systems, Man, and Cybernetics, Part B: Cybernetics, vol. 42, no. 3, pp. 627-646, 2012.

[8] A. Deb, J. S. Roy, and B. Gupta, "Performance comparison of differential evolution, particle swarm optimization and genetic algorithm in the design of circularly polarized microstrip antennas," IEEE Transactions on Antennas and Propagation, vol. 62, no. 8, pp. 3920-3928, 2014.

[9] H. Gao, S. Kwong, B. Fan, and R. Wang, "A hybrid particleswarm Tabu search algorithm for solving job shop scheduling problems," IEEE Transactions on Industrial Informatics, vol. 10, no. 4, pp. 2044-2054, 2014.

[10] L. A. Prashanth and S. Bhatnagar, "Threshold tuning using stochastic optimization for graded signal control," IEEE Transactions on Vehicular Technology, vol. 61, no. 9, pp. 3865-3880, 2012.

[11] F. S. Abu-Mouti and M. E. El-Hawary, "Optimal distributed generation allocation and sizing in distribution systems via artificial bee colony algorithm," IEEE Transactions on Power Delivery, vol. 26, no. 4, pp. 2090-2101, 2011.

[12] T. S. Rappaport, Wireless Communications Principles and Practice, Second Version, Prentice Hall, 2002.

[13] M. Younes, M. Rahli, and L. Abdelhakem-Koridak, "Optimal power flow based on hybrid genetic algorithm," Journal of Information Science and Engineering, vol. 23, no. 6, pp. 18011816, 2007.

[14] E. Vallada and R. Ruiz, "A genetic algorithm for the unrelated parallel machine scheduling problem with sequence dependent setup times," European Journal of Operational Research, vol. 211, no. 3, pp. 612-622, 2011.

[15] L. Chen, A. Langevin, and D. Riopel, "A tabu search algorithm for the relocation problem in a warehousing system," International Journal of Production Economics, vol. 129, no. 1, pp. 147156, 2011.

[16] S. Hanafi, "On the convergence of tabu Search," Journal of Heuristics, vol. 7, no. 1, pp. 47-58, 2001. 


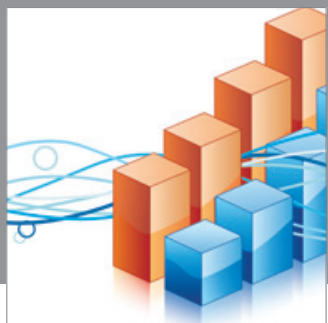

Advances in

Operations Research

mansans

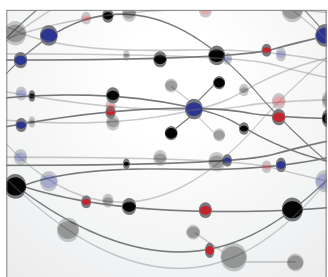

The Scientific World Journal
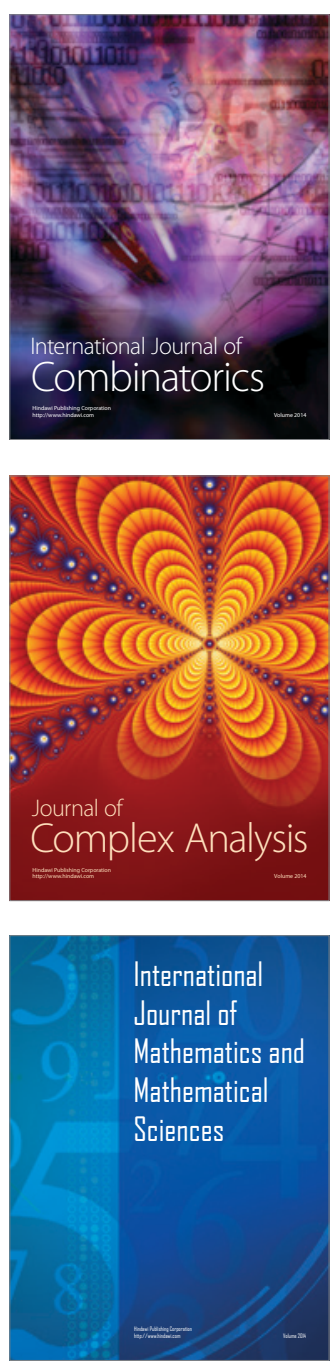
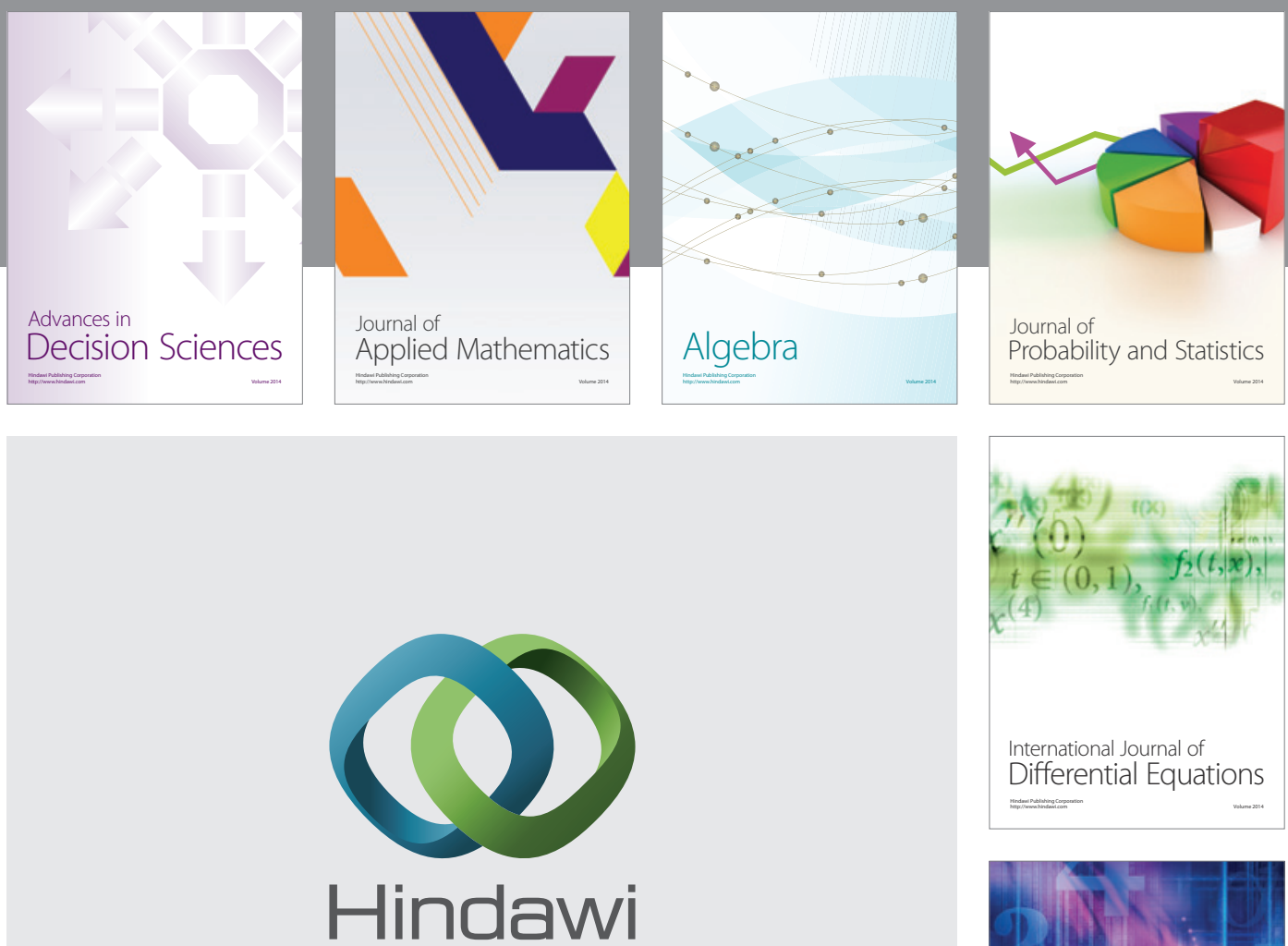

Submit your manuscripts at http://www.hindawi.com
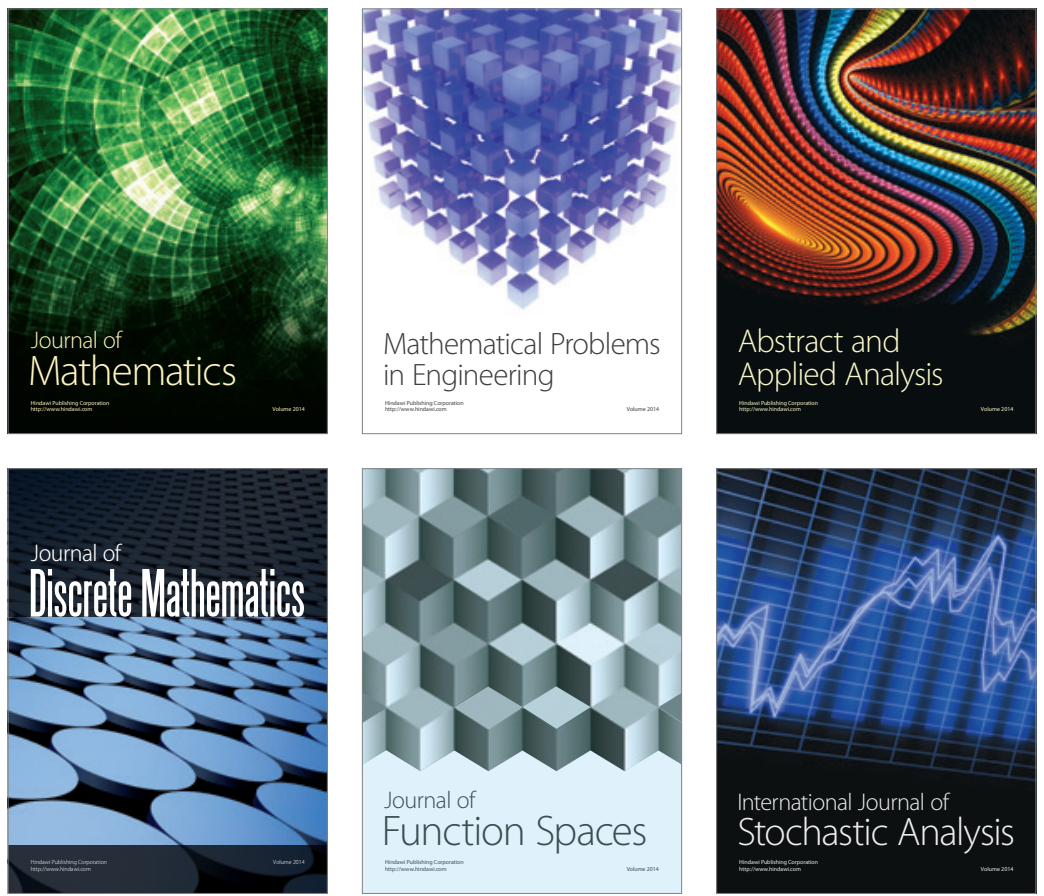

Journal of

Function Spaces

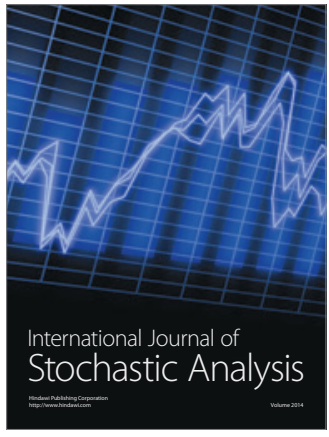

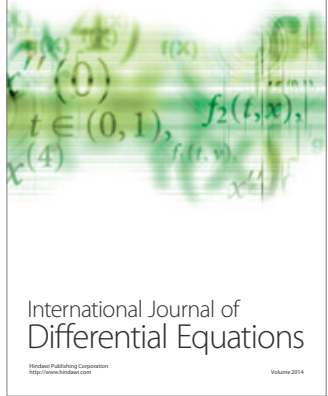
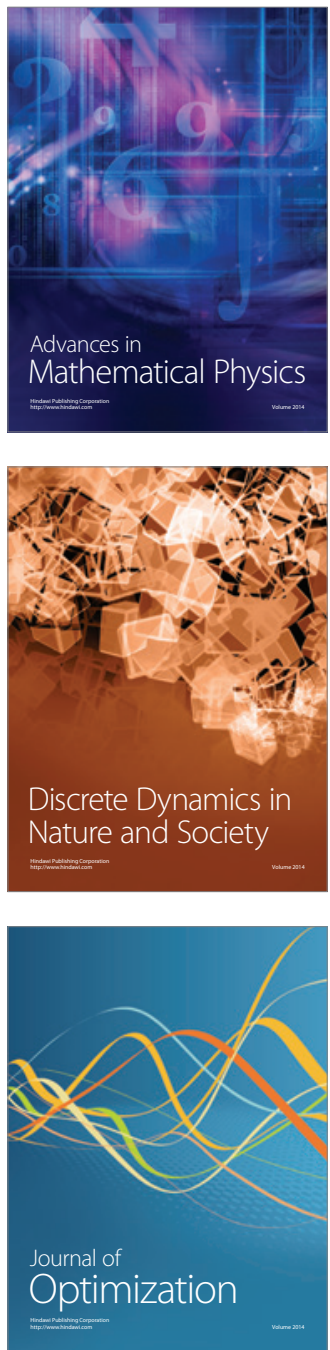